\title{
Políticas Públicas: a importância da aplicabilidade efetiva para detecção precoce da cardiopatia congênita
}

Public Policies: the importance of effective applicability for early detection of congenital heart disease

Políticas públicas: la importancia de la aplicabilidad efectiva para la detección temprana de cardiopatías congénitas

Bruna Eduarda Lopes Felice

ORCID: https://orcid.org/0000-0003-0596-4485 Faculdade de Medicina de São José do Rio Preto, Brasil E-mail: brunafelice4@gmail.com

Alexandre Lins Werneck

ORCID: https://orcid.org/0000-0002-2911-8091 Faculdade de Medicina de São José do Rio Preto, Brasil E-mail: alexandre.werneck@ famerp.br

Daise Lais Machado Ferreira

ORCID: https://orcid.org/0000-0003-4747-5364 Faculdade de Medicina de São José do Rio Preto, Brasil E-mail: daise_lais@yahoo.com.br

\begin{abstract}
Resumo
Introdução: a cardiopatia congênita (CC) é caracterizada por malformações no coração e/ou nos vasos que o constituem, derivados de erros no desenvolvimento e na evolução cardíaca durante a gestação e que permanecem após o nascimento. A Triagem Neonatal é essencial para a prevenção, promoção e recuperação da saúde de neonatos. Objetivo: avaliar se as políticas públicas realizadas de forma efetiva interferem na detecção precoce da cardiopatia congênita. Métodos: pesquisa aprovada pelo Comitê de Ética em Pesquisa da FAMERP. Estudo transversal, delineamento descritivo, abordagem quantitativa-analítica, realizado em um hospital de referência do estado de São Paulo. Foi aplicado um questionário com quatro partes: caracterização; dados em relação ao pré-natal; diagnóstico; conhecimento dos familiares. Critérios de inclusão: pais que se prontificaram a participar da pesquisa. Critérios de exclusão: pais não encontrados para responder ao questionário. Resultados: amostra predominantemente feminina, casadas, residentes na Região Sudeste, idade até 30 anos, um filho, Ensino Médio completo e renda familiar de um salário-mínimo. Percebeu-se que $43,14 \%$ das gestantes foram a seis ou mais consultas no pré-natal, como o preconizado. Em 60,78\% dos neonatos com CC, o diagnóstico aconteceu somente após o nascimento. Houve relatos de mães sobre não haver equipe multiprofissional durante o pré-natal. Conclusão: com profissionais de saúde capacitados e atuação da equipe multidisciplinar, pode-se perceber sinais sugestivos de CC, promover assistência qualificada e detectar precocemente a CC. Considerando os recursos materiais e humanos, é possível iniciar o tratamento no serviço de origem ou encaminhar, inclusive sob recurso de tratamento fora do domicílio (TFD), preconizado pelo Ministério da Saúde.
\end{abstract}

Palavras-chave: Cardiopatia congênita; Detecção precoce; Saúde da criança; Políticas públicas.

\begin{abstract}
Introduction: Malformations in the heart and vessels are derived from errors in cardiac development and evolution during pregnancy and persist after birth. These events characterize congenital heart disease (CC). Neonatal Screening is essential for the prevention, promotion, and health recovery of newborns. Objective: assess whether public policies implemented effectively interfere in the early detection of congenital heart disease. Methods: FAMERP Research and Ethics Committee approved the study. We conducted a cross-sectional study with a descriptive design and quantitative-analytical approach in a reference hospital in the inland of São Paulo State. We formulated a four-part questionnaire with characterization, data regarding prenatal care; diagnosis; knowledge of family members. Inclusion criteria: parents who volunteered to participate in the research. Exclusion criteria: parents not found to answer the questionnaire. Results: predominantly female sample, married, residing in the Southeast Region, aged up to 30 years, one child, completed high school, and a family income of one minimum wage. We noticed that $43.14 \%$ of pregnant women attended six or more prenatal consultations, as recommended. In $60.78 \%$ of neonates with CC, the diagnosis took place only after birth. There are reports from mothers about not having a multidisciplinary team during prenatal care. Conclusion: with trained health professionals and the work of the multidisciplinary team, it is possible to notice
\end{abstract}


signs suggestive of $\mathrm{CC}$, promote qualified care, and detect CC early. It is possible to start the treatment at the origin service or forward it, Considering the material and human resources, even under the home treatment resource (TFD), recommended by the Ministry of Health.

Keywords: Congenital heart disease; Early detection; Child health; Public policy.

\section{Resumen}

Introducción: Las malformaciones en el corazón y los vasos se derivan de errores en el desarrollo y evolución cardíaca durante el embarazo y persisten después del nacimiento. Estos eventos caracterizan la cardiopatía congénita (CC). El cribado neonatal es fundamental para la prevención, promoción y recuperación de la salud de los recién nacidos. Objetivo: evaluar si las políticas públicas implementadas efectivamente interfieren en la detección temprana de cardiopatías congénitas. Métodos: El Comité de Ética e Investigación de la FAMERP aprobó el estudio. Realizamos un estudio transversal con diseño descriptivo y abordaje cuantitativo-analítico en un hospital de referencia del interior del Estado de São Paulo. Se formuló un cuestionario de cuatro partes con caracterización, datos sobre atención prenatal; diagnóstico; conocimiento de los miembros de la familia. Criterios de inclusión: padres que se ofrecieron como voluntarios para participar en la investigación. Criterios de exclusión: padres no encontrados para responder el cuestionario. Resultados: muestra predominantemente femenina, casada, residente en la Región Sudeste, edad hasta 30 años, un hijo, bachillerato completo y un ingreso familiar de un salario mínimo. Observamos que el 43,14\% de las embarazadas acudieron a seis o más consultas prenatales, según lo recomendado. En el 60,78\% de los neonatos con CC, el diagnóstico se realizó solo después del nacimiento. Hay informes de madres sobre no contar con un equipo multidisciplinario durante la atención prenatal. Conclusión: con profesionales de la salud capacitados y el trabajo del equipo multidisciplinario, es posible detectar signos sugestivos de CC, promover una atención calificada y detectar de manera precoz la CC. Es posible iniciar el tratamiento en el servicio de origen o reenviarlo, considerando los recursos materiales y humanos, incluso bajo el recurso de tratamiento domiciliario (TFD), recomendado por el Ministerio de Salud.

Palabras clave: Cardiopatías congénitas; Detección temprana; Salud de los niños; Políticas públicas.

\section{Introdução}

A cardiopatia congênita (CC) é caracterizada por malformações no coração e/ou nos vasos que o constituem, derivados de erros no desenvolvimento e na evolução cardíaca durante a gestação e que permanecem no nascimento (Mineiro, Gurgel, \& Gonçalves, 2011). Desde 1960 preconiza-se a relevância da Triagem Neonatal, pela Organização Mundial da Saúde (OMS), com o objetivo de prevenção, promoção e recuperação da saúde de neonatos (Ministério da Saúde,2002).

A cada 1000 recém-nascidos vivos, dois possuem cardiopatia congênita (CC) e 30\% recebem a alta hospitalar sem diagnóstico. Esse distúrbio cardíaco apresenta-se como responsável por 40\% das mortes por anomalias congênitas, no primeiro ano de vida e, os diagnósticos no pré-natal ocorrem em menos de 50\% (Movahedian, Mosayebi, \&Sagheb, 2016). Dessa forma, ressalta-se a relevância da qualificação nesse período e as políticas públicas que o envolvem, a fim de buscar a detecção precoce, e por sua vez, a minimização da taxa de morbimortalidade neonatal (Sociedade Brasileira de Cardiologia, 2011).

Nos Estados Unidos, estima-se que 44,5\% dos neonatos com CC falecem ainda no primeiro ano de vida. Na América Latina, a segunda principal causa de morte em crianças, com idade inferior a um ano, é designada a cardiopatia congênita(Belo, Oselame, \& Neves, 2016; Frota, Andrade, Santos, Silva, \& Fernandes, 2014). No Brasil, partindo do pressuposto de que há o registro anual de 2,8 milhões de nascidos vivos, estima-se que surjam em média 29 mil novos casos por ano de cardiopatia congênita. Além disso, percebe-se que há maior incidência de anomalias congênitas na Região Sudeste, se comparado com as demais regiões, conforme os dados do Sistema de Informações sobre Nascidos Vivos (SINASC) (Ministério da Saúde, 2017).

Nos primeiros dias de vida, alguns recém-nascidos podem não apresentar sinal/sintoma sugestivo de cardiopatia congênita. Desse modo, faz-se necessário um exame físico de qualidade para detectar possíveis alterações e impedir a alta hospitalar sem o diagnóstico prévio (Movahedianet al., 2016). Dessa maneira, salienta-se a importância de agilidade no diagnóstico precoce, e posteriormente, o início do tratamento, buscando impedir maiores sequelas futuras no neonato (Canal 
Saúde, 2019), evitando o comprometimento cardiovascular, lesões em outros órgãos, e até o óbito (Movahedianet al., 2016; Chamsi-Pasha, \&Chamsi-Pasha, 2016).

A detecção precoce faz-se necessária no âmbito da cardiopatia congênita, justificando a implementação efetiva das políticas públicas estabelecidas pelo Ministério da Saúde, como a triagem pré-natal e neonatal. Para que isto ocorra, são imprescindíveis profissionais capacitados e olhar clínico ampliado, resultando na melhoria do prognóstico de neonatos e crianças, minimizando as sequelas, dando oportunidade de vida e possibilitando à família maior conhecimento sobre o assunto. Ressalta-se a importância do diagnóstico precoce da cardiopatia congênita precocemente, logo no pré-natal, como forma de auxiliar na decisão pelo nascimento em um hospital com suporte e assistência adequados, oferecendo promoção, prevenção e recuperação da saúde. O objetivo deste estudo foi avaliar se as políticas públicas realizadas de forma efetiva interferem na detecção precoce da cardiopatia congênita.

\section{Metodologia}

Estudo transversal, com delineamento descritivo, abordagem quantitativa do tipo analítica, com correlação entre as variáveis "O diagnóstico aconteceu em qual momento" e "Realizou quantas consultas de pré-natal". A população do estudo foi composta por 51 pais de crianças com cardiopatia congênita atendidas no Hospital Campo de Estudo, cujos pais estavam presentes no momento da coleta de dados e aptos a responderem ao questionário. A amostra foi por amostragem não probabilística em sequência, ou seja, incluímos todos os pais das crianças com cardiopatia congênita, cujos filhos apresentavam idade igual ou menor do que cinco anos, internadas no período de novembro de 2020 a fevereiro de 2021 e que aceitaram participar da pesquisa. Os critérios de exclusão foram os pais que não estavam presentes na unidade de internação nos horários estipulados para coleta de dados.

Esta pesquisa foi aprovada pelo Comitê de Ética em Pesquisa da Faculdade de Medicina de São José do Rio Preto (FAMERP), Parecer $\mathrm{n}^{\mathrm{o}}$ 4027642, de 14/05/2020. Todos os participantes assinaram o Termo de Consentimento Livre Esclarecido, conforme exige a Resolução no. 466, de 12 de dezembro de 2012, do Conselho Nacional de Saúde, que rege as pesquisas envolvendo seres humanos. Assim como obedeceu a todos os preceitos éticos legais, que envolve as pesquisas com seres humanos.

O instrumento de coleta de dados foi elaborado pela pesquisadora e contêm quatro partes, sendo elas: $1^{\circ}$ parte Caracterização (11 perguntas de múltipla escolha); $2^{\circ}$ parte - Dados em relação ao pré-natal (seis perguntas de múltipla escolha); $3^{\circ}$ parte - Diagnóstico (cinco perguntas de múltipla escolha); $4^{\circ}$ parte - Conhecimento dos familiares (12 perguntas de múltipla escolha) contemplando as variáveis do estudo. A entrevista ocorreu nos períodos, matutino e vespertino, respeitando a disponibilidade dos participantes, durante em média de cinco a dez minutos. As entrevistas foram realizadas no tempo livre da pesquisadora, no próprio quarto em que as crianças estavam internadas. Todos os cuidados foram tomados pela pesquisadora para garantir o anonimato e a segurança dos respondentes. O local de coleta foi em um Hospital de referência no Brasil em assistência, ensino e pesquisa, localizado no estado de São Paulo.

Após a tabulação dos dados coletados, foram exercidas duas funções de análises estatísticas: descritiva e inferencial. De maneira descritiva, foi traçado o perfil da amostra estudada, contemplando as variáveis analisadas e seus desdobramentos. Os dados foram replicados de forma absoluta e relativa, nesta primeira parte. No âmbito inferencial, foi traçado como objetivo estatístico, a análise de *dependência* e predição entre as variáveis propostas no escopo do trabalho. Foi executado o teste de normalidade de KolmogorovSmirnov. Os resultados *da dependência* entre as variáveis propostas foram obtidos por meio de análise entre os valores de P (significância). Todas as análises foram obtidas por meio do software SPSS Statistics ${ }^{\circledR}$ (versão 23), atrelado às funcionalidades da ferramenta Microsoft Excel ${ }^{\circledR}$ (versão 2016). 
Para descrição dos resultados foram calculadas as frequências relativas e absolutas, média, desvio padrão e mediana. Para conhecer os desfechos das variáveis não paramétricas foi aplicado o teste U de Mann-Whitney, para análise do comportamento das correlações entre as variáveis analisadas e o grau de explicação da variável dependente em relação das variáveis independentes da amostra. O resultado foi considerado significativo quando $\mathrm{p}<0,05$.

\section{Resultados}

A amostra $(n=51)$ desta pesquisa foi constituída em maioria por mulheres casadas, que residem na Região Sudeste, com idade de até 30 anos, com um filho, Ensino Médio completo e renda familiar de um salário-mínimo.

Tabela 1. Caracterização sociodemográfica da amostra. São José do Rio Preto, SP, Brasil. 2020.

\begin{tabular}{|lcc|}
\hline Estado Civil & N & \% \\
\hline Solteiro(a) & 17 & 33,33 \\
Casado(a) & 27 & 52,94 \\
\hline Região & 26 & 50,98 \\
\hline Sudeste & & 66,67 \\
\hline Idade & 34 & 33,33 \\
\hline Até 30 anos & & \\
\hline Escolaridade & 17 & \\
Ensino Médio Completo & \\
\hline Renda & 22 & 43,14 \\
\hline 1 Salário-Mínimo & \\
\hline
\end{tabular}

Foram retirados da tabela, os dados que apareceram com menor relevância. Fonte: Autores (2020).

Tabela 2. Dados em relação ao pré-natal, Diagnóstico e Conhecimento dos familiares. São José do Rio Preto, SP, Brasil. 2020.

\begin{tabular}{|c|c|c|}
\hline Após o diagnóstico houve mudanças na renda? & $\mathbf{N}$ & $\%$ \\
\hline Sim & 33 & 64,71 \\
\hline \multicolumn{3}{|l|}{ Se sim, aumentou ou diminuiu? } \\
\hline Diminuiu & 32 & 62,75 \\
\hline \multicolumn{3}{|l|}{ Que recursos utilizou } \\
\hline TFD & 26 & 50,98 \\
\hline \multicolumn{3}{|l|}{ A partir de qual mês de gestação iniciou o pré natal? } \\
\hline 1 a 3 meses & 30 & 58,82 \\
\hline \multicolumn{3}{|l|}{ Realizou quantas consultas durante o pré-natal? } \\
\hline Mais de 6 Consultas & 22 & 43,14 \\
\hline \multicolumn{3}{|c|}{$\begin{array}{l}\text { Foi acompanhada/atendida pelo mesmo médico durante todo o pré- } \\
\text { natal? }\end{array}$} \\
\hline Sim & 37 & 72,55 \\
\hline \multicolumn{3}{|l|}{\begin{tabular}{|l} 
Havia equipe multiprofissional? \\
\end{tabular}} \\
\hline Não & 24 & 47,06 \\
\hline \multicolumn{3}{|l|}{ O diagnóstico aconteceu em qual momento? } \\
\hline Após o nascimento & 31 & 60,78 \\
\hline \multicolumn{3}{|l|}{ O diagnóstico foi feito por meio de } \\
\hline Consultas/exames de rotina & 30 & 58,82 \\
\hline \multicolumn{3}{|l|}{ Qual plano terapêutico foi inserido? } \\
\hline Cirúrgico & 39 & 66,10 \\
\hline \multicolumn{3}{|l|}{ Qual/Quais profissionais te explicaram? } \\
\hline Médico & 40 & 76,92 \\
\hline \multicolumn{3}{|c|}{$\begin{array}{l}\text { Você sabe qual a importância de fazer o acompanhamento com } \\
\text { profissionais da saúde durante o período pré-natal e pós-natal? }\end{array}$} \\
\hline Sim & 45 & 88,24 \\
\hline \multicolumn{3}{|l|}{ Se sim, qual a importância? } \\
\hline Diagnóstico precoce & 22 & 37,29 \\
\hline
\end{tabular}


Research, Society and Development, v. 10, n. 11, e56101119371, 2021

(CC BY 4.0) | ISSN 2525-3409 | DOI: http://dx.doi.org/10.33448/rsd-v10i11.19371

\begin{tabular}{|l|c|c|}
\hline Cuidados com a saúde do bebe & 23 & 38,98 \\
\hline Quem te explicou a importância? & & \\
\hline Médico & 44 & 67,69 \\
\hline $\begin{array}{l}\text { Quais foram os sinais que te orientaram que poderiam causar risco } \\
\text { ao bebê? }\end{array}$ & & \\
\hline Cianose/roxinho & 15 & 22,39 \\
\hline Não houve orientação & 16 & 23,88 \\
\hline
\end{tabular}

Foram retirados da tabela, os dados com menor relevância. Fonte: Autores (2020).

Por meio da análise do cruzamento inferencial, aplicando-se o teste de Correlação Mann-Whitney, conclui-se que as variáveis "O diagnóstico aconteceu em qual momento" e "Realizou quantas consultas de pré-natal" estão estatisticamente correlacionadas $(\mathrm{p}=0,007)$.

\section{Discussão}

Nesta pesquisa, a amostra foi constituída por 51 mulheres/mães, sendo que 33,33\% são solteiras e 52,94\% casadas, o que corrobora o estado civil de outro estudo, cuja população foi de dez mulheres, sendo que 90\% eram casadas (Menezes, Porto, Rodrigues, Oliveira, \& Marques, 2020). A discrepância entre as amostras, pode ser explicada, pelo tipo de estudo, uma vez que, a atual pesquisa é quantitativa e o estudo correlacionado é qualitativo. Dessa forma, ressalta-se a importância de um apoio biopsicossocial para essas mães, visto que, desde o momento do diagnóstico da cardiopatia congênita, até o procedimento e a recuperação, há um processo intenso de readaptação e assimilação de todas as mudanças e descobertas.

Além disso, na presente pesquisa, constatou-se que 50,98\% são procedentes da Região Sudeste, 19,61\% da Região Norte e 19,61\% da Região Centro-Oeste, resultados que divergem de um estudo, no qual a amostra corresponde a 14 mães e, dentre elas, 78,6\% são oriundas da região Norte (Cesario, Carneiro, \& Dolabela, 2020). Ressalta-se que, na presente pesquisa, foi realizada uma abordagem quantitativa, enquanto que na pesquisa citada anteriormente tratou-se de um estudo qualitativo. A divergência de resultados pode ser explicada em decorrência das localizações diferentes, uma vez que, a pesquisa atual foi desenvolvida em um hospital na cidade de Belém (PA).

Em relação ao questionamento, "Quais recursos foram utilizados para se deslocar até o Hospital", no caso de famílias que não residem na cidade, 50,98\% relataram que utilizaram o recurso Tratamento fora do Domicílio (TFD). Essa política caracteriza-se como uma política que, visa garantir o tratamento de casos de média e alta complexidade para pacientes que possuem a Cardiopatia, mas que não são tratáveis no local em que residem, pela escassez de recursos e serviços especializados. Além disso, o TFD oferece as passagens de ida e volta, para os familiares, tratamentos desde ambulatoriais até cirúrgicos, entre outros. Dessa forma, é possível constatar de que a política instituída pelo Ministério da Saúde para a Atenção de Gestantes de Alto Risco está sendo realizada de forma efetiva, proporcionando o cuidado e atenção adequada às crianças e familiares, além de permitir a possibilidade de detecção precoce, independentemente do local (Secretaria de Saúde do Estado do Piauí, 2017).

Em um levantamento realizado pelo Ministério da Saúde, sobre hospitais que desenvolvem ações de detecção precoce das doenças cardiovasculares, identificou-se que na Região Norte, entre três hospitais, dois (67\%) desenvolvem ações de detecção precoce. Na região Nordeste,entre 14 hospitais, dez (71\%) realizam atividades voltadas para esse objetivo. E por fim, na Região Sudeste, de dezenove hospitais, dezesseis (84\%) desenvolvem ações com a mesma finalidade (PROADIS-SUS, 2020). Diante deste achado, salienta-se que na Região Sudeste há maior oportunidade de detecção precoce, e isto, pode ser explicado frente às diversas tecnologias e equipamentos que propiciam resultados de exames mais fidedignos. Dessa forma, faz-se um questionamento se as mães que são de outras regiões vieram em um momento oportuno para a detecção precoce da 
cardiopatia congênita. É imprescindível a necessidade que as políticas públicas independam de recursos avançados e da região em que estão sendo realizadas.

$\mathrm{Na}$ atual pesquisa, a amostra foi constituída 66,67\% por mulheres com idade até 30 anos e, 27,45\%, com idades entre 31 e 50 anos, o que corrobora um estudo, cuja população era de 117 mães, com idade média de 29 anos (Bolaséllet al., 2019). Entende-se que o amadurecimento é um fator de extrema relevância para lidar e entender as situações e contextos, mas, por outro lado, sabe-se que a gestação com idade avançada pode levar a uma gestação de risco e, consequentemente, compromete a saúde do feto. Diante desse cenário, há a necessidade de um processo humanizado desde o pré-natal, com intensa comunicação e diálogo entre a equipe e os familiares, a fim de promover maior adesão a todas as etapas e propiciar melhor compreensão por parte dos familiares, seja em situações esperadas ou não esperadas. Isto feito, há a possibilidade de promover a deteç̧ão precoce e minimizar maiores complicações para o recém-nascido e transtornos aos familiares.

Em relação ao questionamento: “A partir de qual mês de gestação iniciou o pré-natal?”, 58,82\% relataram o início entre o $1^{\circ}$ e $3^{\circ}$ mês. Além disso, 43,14\% da amostra relataram ter realizado mais de seis consultas no pré-natal. Em contrapartida, 60,78\% disseram que o diagnóstico ocorreu após o nascimento, o que diverge dos dados apresentados anteriormente. Dessa forma, quando foi realizada a análise inferencial com correlação entre as variáveis "O diagnóstico aconteceu em qual momento $X$ Realizou quantas consultas durante o pré-natal",o valor foi de 0,007, concluindo que as variáveis analisadas, a partir do método de Mann-Whitney, estão estatisticamente correlacionadas. Não foram encontrados estudos que corroborassem os resultados encontrados.

Diante dos dados apresentados, é importante ressaltar que houve adesão ao pré-natal por parte dos familiares, porém, entende-se que os procedimentos não foram realizados de forma efetiva para que fossem descobertos precocemente. Dessa forma, ressalta-se que diagnóstico deve ser realizado ainda no pré-natal, visto que há exames que fazem parte desse processo e que possibilitam a detecção precoce, se executado de forma correta e efetiva, como o exame de Ultrassonografia, que deve ser feito no $1^{\circ}$ e $2^{\circ}$ trimestres de gestação (Portaria n. 1.727, 2017; Pedra et al., 2019).

Proporciona-se, assim, uma reflexão sobre quais foram as lacunas no processo que implicaram nesses resultados, pois: 47,06\% da amostra relataram que não havia uma equipe multidisciplinar, o que pode estar diretamente relacionado com a dificuldade na detecção precoce. De acordo com o Ministério da Saúde, a equipe multidisciplinar possui papel fundamental para propor decisões coerentes e assistência de qualidade aos neonatos e crianças portadoras da Cardiopatia Congênita (Portaria n. 1.727, 2017). Além disso, quando há a atuação da equipe multidisciplinar e interdisciplinar trabalhando em conjunto, as possibilidades de detectar precocemente a cardiopatia congênita aumentam, visto que, experiências e visões diferentes somadas, podem gerar um resultado mais efetivo para diagnósticos tão complexos.

A capacitação e supervisão dos profissionais auxiliará no aperfeiçoamento da visão ampliada, diante de exames realizados durante todo o período gestacional e após o parto, assim como de sinais sugestivos de cardiopatia congênita, gerando como consequência, uma maior facilidade de detecção precoce. Além disso, a detecção precoce minimiza um conjunto de sentimentos e aflições dos familiares, garantindo um bom prognóstico e maiores chances de qualidade de vida para essa criança futuramente.

A partir do questionamento sobre, "Quem te explicou a importância do pré-natal", 67,69\% relataram ter sido o médico e apenas 10,77\% disseram que foi o enfermeiro, o que diverge de uma revisão integrativa de literatura, cuja pesquisa selecionou 10 publicaçõessobre o papel do enfermeiro no acompanhamento do pré-natal e demonstrou que a atuação dos enfermeiros no pré-natal sendo aperfeiçoada ao longo dos últimos anos, ou seja, com a melhoria dos vínculos, refletindo de forma positiva em toda a gestação. Porém, o estudo relatou que ainda há carência do reconhecimento do enfermeiro na sociedade sobre o papel que desempenha no pré-natal (Silva, \& Andrade, 2020). 
A enfermagem atua na promoção, prevenção e recuperação da saúde, assim como, auxilia na deteç̧ão precoce. Todos estes feitos contribuem para um prognóstico oportuno à criança, que refletem de forma positiva aos familiares, no que diz respeito ao cuidado propriamente dito, como também nas formas de enfrentamento e entendimento sobre a cardiopatia congênita e os cuidados inerentes a ela (Assunçãoet al., 2020).

Um estudo qualitativo, cuja amostra foi de 15 gestantes, corrobora os achados anteriores, visto que, identificou-se que $60 \%$ de sua amostra não possuíam conhecimento de que o enfermeiro pode realizar o pré-natal, com valorização apenas da figura médica, reafirmando a falta de reconhecimento sobre o enfermeiro ser um profissional apto a realizar essa função (Meloet al., 2021). Dessa forma, o enfermeiro que realiza os cuidados do pré-natal com excelência, favorece a valorização e necessidade deste profissional no pré e no pós-natal, a fim de colaborar na diminuição das taxas da morbimortalidade infantil e materna (Benedetet al., 2021).

Em relação ao questionamento, "Quais foram os sinais que te orientaram que poderiam causar risco ao bebêe", 23,39\% relataram cianose/roxinho e 23,88\% disseram que não houve orientação. Não foi possível encontrar estudos que corroborassem estes achados. Por outro lado, é preciso enfatizar que os pais das crianças que possuem cardiopatia congênita, devem ser orientados pelos profissionais da saúde quanto aos fatores preventivos, profiláticos, de imunização e nutrição, a fim de proporcionar um bom prognóstico (Bertoletti, Marx, Hattge Júnior, \& Pellanda, 2014).

\section{Conclusão}

É possível concluir que, a partir de profissionais de saúde capacitados e da atuação das equipes multidisciplinar e interdisciplinar, pode-se perceber sinais e sintomas sugestivos de cardiopatia congênita, promover uma assistência qualificada e, posteriormente, detectar precocemente a CC. A partir disto, considerando os recursos materiais e humanos, é possível iniciar o tratamento no serviço de origem ou encaminhar, inclusive sob recurso de tratamento fora do domicílio (TFD), preconizado pelo Ministério da Saúde.

Ressaltamos que a pandemia foi considerada um fator limitante para a pesquisa, por conta da restrição de horários para a coleta de dados. Percebemos um grande potencial no estudo, que consiste em uma proposta de reflexão por parte dos gestores de saúde em nível municipal, com possibilidade de avaliar e se necessário, adequar as ações contempladas nas políticas públicas.

\section{Referências}

Assunção, C. S., Rizzo, E. R., Santos, M. E., Carvalho, J. B., Basílio, M. D., \& Messias, C. M. (2020). The Nurse in PrenatalCare: The PregnantWomen. Revista De Pesquisa Cuidado é Fundamental Online, 11(3), 576-581. https://doi.org/10.9789/2175-5361.2019.v11i3.576-581

Belo, W. A., Oselame, G. B., \& Neves, E. B. (2016). Perfil clínico-hospitalar de crianças com cardiopatia congênita. Cadernos de Saúde Coletiva, 24(2), 216220. http:// doi.org/10.1590/1414- 462X201600020258

Benedet, D. C. F., Wall, M. L., Lacerda, M. R., Machado A. V. M. B., Borges, R., \&Zômpero, J. F. J. (2021). Strengthening nurses in prenatal care through reflection-action. RevistaGaúcha de Enfermaggem, 42, e20200187. https://doi.org/10.1590/1983-1447.2021.20200187

Bertoletti, J., Marx, G. C., HattgeJúnior, S. P., \&Pellanda, L. C. (2014). Quality of Life and Congenital Heart Disease in Childhood and Adolescence.Arquivos Brasileiros de Cardiologia, 102(2), 192-198. https://doi.org/10.5935/abc.20130244

Bolaséll, L. T., Foschiera,. N., Luft, C. Z., Crestani, P. L., Woinarovicz, B., Silva, . F., \& Schneider, C. (2019). Caracterização de mães de crianças cardiopatas congênitas internadas em uma UTI pediátrica. Psicologia Hospitalar, 17(1), 17-33. Recuperado de http://pepsic.bvsalud.org/pdf/ph/v17n1/17n1a03.pdf

Canal Saúde. (2019). Ministério da Saúde reforça a importância do Teste do Pezinho entre o $3^{o}$ e $5^{o}$ dia de vida. https://www.canalsaude.fiocruz.br/noticias/noticiaAberta/ministerio-da-saude-reforca-a-importancia-do-teste-do-pezinho-entre-o-3o-e-5o-dia-devida06062019

Cesario, M. S. A., Carneiro, A. M. F., \& Dolabela, M. F. (2020). Mães de crianças com cardiopatia congênita: dúvidas e estratégia de intervenção. Revista Eletrônica Acervo Saúde, 12(5), e2337.https://doi.org/10.25248/reas.e2337.2020 
Research, Society and Development, v. 10, n. 11, e56101119371, 2021

(CC BY 4.0) | ISSN 2525-3409 | DOI: http://dx.doi.org/10.33448/rsd-v10i11.19371

Chamsi-Pasha, M. A., \&Chamsi-Pasha, H. (2016). Critical congenital heart disease screening.AvicennaJournalof Medicine, 6(3), 65-68. http:// doi.org/10.4103/2231-0770.184062

Frota, M. A., Andrade, I.S., Santos, Z. M. S. A., Silva, C. A. B., \& Fernandes, A. F. C. (2014). Perfil sociodemográfico familiar e clínico de crianças com cardiopatia congênita atendidas em uma instituição hospitalar. Revista Brasileira em Promoção à Saúde, 27(2), 239-246. http:// doi.org/10.5020/18061230.2014.p239.

Melo, L. D., Araújo, A. B., Teixeira, L. G., Santos, L. R., Pereira, R. J., Fernandes, M. T. A. C. N., Affonso, D. L. S., Rosendo, A. A., Silva, P. H. B., \& Taroco, F. E. (2021). Intensive care for congenital heart diseases: Notes on neonatal nursing care. Research, Society and Development, $10(5)$, e52310515346. https://doi.org/10.33448/rsd-v10i5.15346

Menezes, L. T., Porto, M. A., Rodrigues, D. G., Oliveira, J. A. S., \& Marques, H. S. (2020). Vivência de mães de crianças com cardiopatia congênita que serão submetidas à cirurgia cardiovascular. Revista da SBPH, 23(1), 134-146. http://pepsic.bvsalud.org/pdf/rsbph/v23n1/12.pdf

Mineiro, J. C., Gurgel, J. M. S., \& Gonçalves, R. L. (2011). Cardiopatias Congênitas: uma visão geral. Periódico Científico do Núcleo de Biociências - Centro Universitário Metodista Izabela Hendrix, 1(2), 62-81. https://www.metodista.br/revistas/revistas-izabela/index.php/bio/article/view/241/217

Ministério da Saúde. (2002). Manual de Normas Técnicas e Rotinas Operacionais do Programa Nacional de Triagem Neonatal. Ministério da Saúde, Brasília, DF, Brasil. http://bvsms.saude.gov.br/bvs/publicacoes/triagem_neonatal.pdf

Ministério da Saúde. (2017). Síntese de evidências para políticas de saúde: diagnóstico precoce de cardiopatas congênitas. Ministério da Saúde, Brasília, DF, Brasil. http://bvsms.saude.gov.br/bvs/publicacoes/sintese_evidencias_politicas_cardiopatias_congenitas.pdf

Movahedian, A. H., Mosayebi, Z,\&Sagheb, S. (2016). Evaluation of Pulse Oximetry in the Early Detection of Cyanotic Congenital Heart Disease in Newborns.Journal of Tehran Univesity Heart Center, 11(2), 73-78. https://www.ncbi.nlm.nih.gov/pmc/articles/PMC5027164/

Pedra, S. R. F. F., Zielinsky, P., Binotto, C. N., Zielinsky, P., Binotto, C. N., Martins, C. N., Fonseca, E. S. V. B., Guimarães, I. C. B., Corrêa, I. V. S., Pedrosa, K. L. M., Lopes, L. M., Nicoloso, L. H. S., Barberato, M. F. A, \&Zamith, M. M. (2019). Diretriz Brasileira de Cardiologia Fetal-2019. ArquivosBrasileiros de Cardiologia, 112(5), 600-648. http:// doi.org/10.5935/abc.20190075

Portaria $n^{o}$ 1.727, de julho de 2017. Aprova o Plano Nacional de Assistência à Criança com Cardiopatia Congênita. https://bvsms.saude.gov.br/bvs/saudelegis/gm/2017/prt1727_12_07_2017.html

PROADIS-SUS. (2020). Projeto Diagnóstico Situacional dos Serviços de Cirurgia Cardíaca Pediátrica habilitados no Sistema Único de Saúde em território nacional. $\quad$ https://www2.camara.leg.br/atividade-legislativa/comissoes/comissoes-temporarias/externas/56a-legislatura/enfrentamento-pandemia-covid19/apresentacoes-em-eventos/WilsonLuiz.pdf

Secretaria de Saúde do Estado do Piauí. (2017). Manual referente à concessão do auxílio para tratamento fora de domicílio - TFD no Sistema Único De Saúde -SUS/PI. http://www.saude.pi.gov.br/ckeditor_assets/attachments/860/manual_tfd_alterado_para_cib_2017.pdf

Silva, A. A. B., \& Andrade, C. (2020). The role of nurses in prenatal care, education and health promotion.Research, Society and Development, 9(10), e9989109477. http://doi.org/10.33448/rsd-v9i10.9477

Sociedade Brasileira de Cardiologia. (2011). Diagnóstico precoce de cardiopatia congênita crítica: oximetria de pulso como ferramenta de triagem neonatal. http://www.sbp.com.br/pdfs/diagnostico-precoce-oximetria.pdf 